\title{
Induction and Migration of Cryptic/Defective Salmonella enterica Prophages as a Consequence of Infection with Lytic Phages Is an Additional Factor in Stability of a Coevolutionary Vector
}

\author{
E. A. Pleteneva ${ }^{a}$, O. V. Shaburova ${ }^{a}$, J. Azeredo $^{b}$, and V. N. Krylov ${ }^{a}$ \\ ${ }^{a}$ State Research Institute of Genetics and Selection of Industrial Microorganisms, Moscow, 117545 Russia; \\ e-mail: krylov@genetika.ru \\ ${ }^{b}$ Universidade do Minho, 4710-057 Braga, Portugal; \\ e-mail:jazeredo@deb.uminho.pt \\ Received October 16, 2008
}

\begin{abstract}
The influence of infection of natural isolates of Salmonella enterica with lytic (nonlysogenic) phages on the expression of resident cryptic or defective prophages in host bacteria was studied. The induction of defective/cryptic phages after infection with nonlysogenic phages and packaging of bacterial chromosomal fragments in capsids of defective phages is demonstrated. This may lead to migration and wide distribution of both the genomes of defective phages per se and various fragments of the bacterial chromosome (including pathogenic islands) in new bacterial strains with concomitant change of their properties, the acquired new features of pathogenicity among them.
\end{abstract}

DOI: $10.1134 / \mathrm{S} 1022795410040174$

One of the serious problems of modern medicine is an increase in the frequency of outbreaks of salmonellosis with new symptomatics caused by Salmonella enterica strains resistant to many antibiotics. It cannot be ruled out that the employment of specific bacteriophages may provide a useful alternative to antibiotic therapy in treatment and prophylaxis of these diseases [1-4]. However, the significance of risks from the introduction of a large number of bacteriophages in treatment and prophylaxis remains unclear: whether or not this should lead to a disturbance of the balance already established in relationships between bacteria and their phages, in particular, to an increase in the frequency of phage migration among various salmonella species. These apprehensions are connected with evidence that bacteriophages participate in the transfer of genes, which determine pathogenicity and virulence, leading to the appearance of pathogens with novel properties [5-8].

We used 31 strains of Salmonella enterica bacteria (25 strains were isolated in Portugal and 6 in Bristol, Great Britain). For differetiation of the greater part of phages, strains Ex2 and 9510.85 (Bristol) were optimal. A total of 22 bacteriophages were selected for this work: phi12, phi31tu, phi38, phi39, phi68, phi169, phi2/2, phiS151, phiA1, phiN4, phiN5, phi45, phi81, phi11, phi2a, phi2b, phi4/1a, phi4/1b, phi135lar, phi135sm, phiRBSc1, and phiRBStu. The phages involved in this work were isolated in Regensburg (Bavaria) in sewages, some phages were isolated in Portugal from various sources, as well as in Moscow from the river Yauza. Bacteriophages were chosen in this work for the trait of inability to form stable lysogens. The last four pairs of phages listed have similar numbers but differ in the appearance of produced plaques $(\mathrm{a} / \mathrm{b}, \mathrm{lar} / \mathrm{sm}, \mathrm{cl} / \mathrm{tu})$. A pair of phages phiRBScl/ phiRBStu (isolated in Regensburg from different samples) proved to be nonrelated (see below). Phages of the remaining three pairs manifested relatedness.

Standard nutrient LB media (liquid and agar) were used [9]. Standard methods of working with phages were as in [10]. Methods for phage concentration, purification by centrifugation in the $\mathrm{CsCl}$ density gradient, DNA isolation, restriction with endonucleases, electrophoresis, and Southern hybridization were as in [11].

We determined homology of DNAs in some phages (dot-blot) (data not shown), compared genome sizes, and elucidated the character of digestion with various enzymes (Figs. 1 and 2), which allows combining 19 of 22 original phages into five groups that are believed to be equivalent to phage species (we did not succeed in classifying three phages):

(1) phi169, phi68, phi39, phi135 (lar and sm), phi38, phi2 (a and b), phi45, phi11, phi81 (DNA homology, similarity of restriction profiles, identicalgenome size);

(2) phi4/1 (a and b); phiRBSc1 (DNA homology);

(3) phi31tu, phiN5, phiA1 (resistant to EcoRV and EcoRI, lack of homology with other phages);

(4) phiRBStu; 


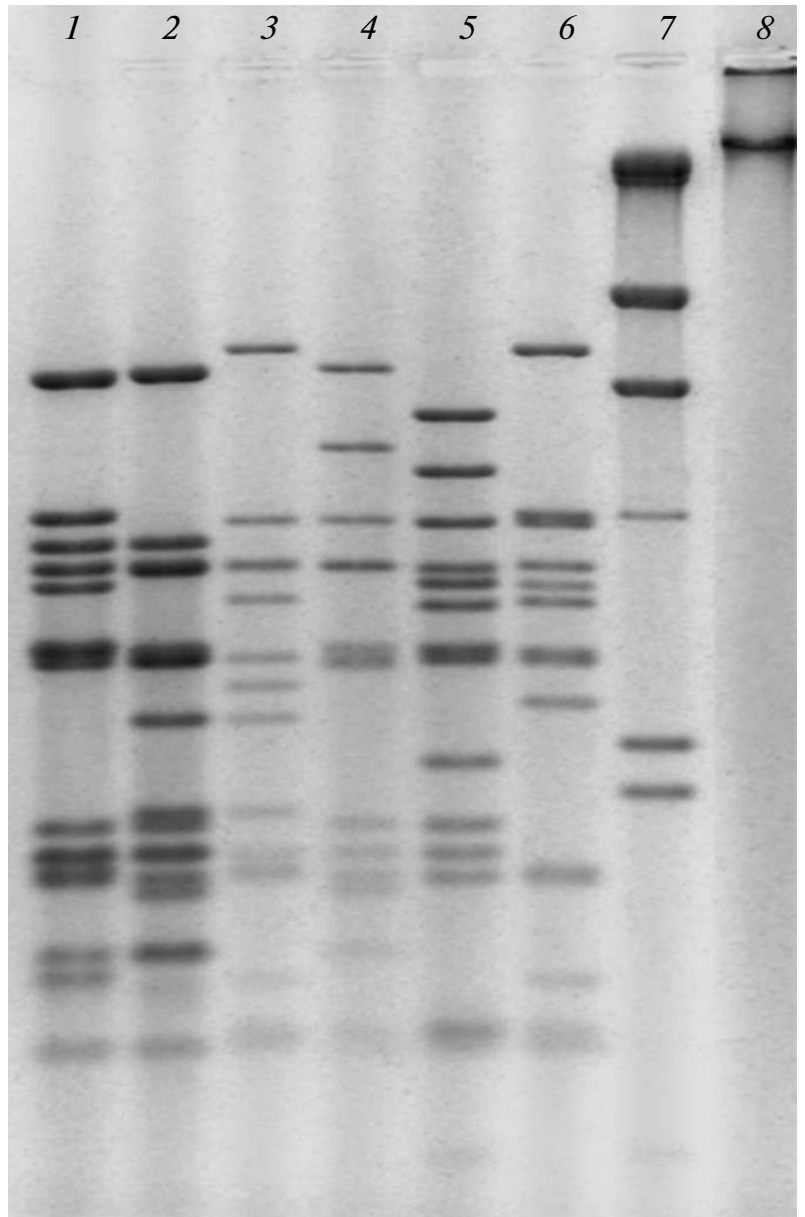

Fig. 1. Digestion of salmonella phage DNA with the enzyme EcoRV. 1, phi135lar (34.7/38.95 kb); 2, phi135sm $(32.35 / 37.66 \mathrm{~kb}) ; 3$, phi387 (33.35 kb); 4, phi169 $(33.78 / 37.43 \mathrm{~kb}) ; \quad 5$, phi68 $(38.83 \mathrm{~kb}) ; \quad 6$, phi39 $(32.09 / 39.09 \mathrm{~kb})$ (in brackets is given the total size of fragments - genome size, $\mathrm{min} / \mathrm{max}$, taking into account double bands); 7, phage $\lambda$ DNA digested with HindIII (fragment size marker); 8 , phi31tu.

(5) phi2/2 (the largest genome, lack of homology with other phages).

In this work, we did not intend to compare newly isolated phages with the currently known species: P27like (phage ST64B); P2-like (Fels-2, SopEphi, and PSP3); lambdoid phages (Gifsy-1, Gifsy-2, Fels-1); P22-like (epsilon34, ES18, P22, ST104, ST64T); T7like (SP6), and three individual species (epsilon15, KS7, and FelixO1) represented by single phages [12], although, as judged by the genome sizes, some of phages isolated in this work may belong to the already identified species. The testing of the variants revealed that neither of examined bacterial strains liberate phages that produce visible plaques in cross-assays. However, during purification of phages by replatig them from single plaques, we did not succeed in obtaining homogeneous (judjing by the appearance of plaques) progeny in some cases. Note that the typical

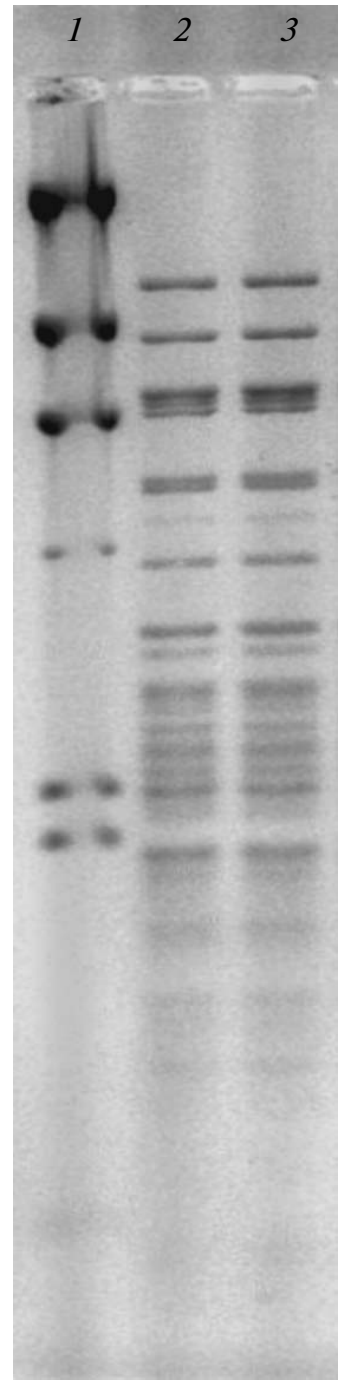

Fig. 2. Digestion with the enzyme HindIII of DNA from phage phi $2 / 2$ obtained on various hosts. $1, \lambda$ HindIII (fragment size marker); 2, phi2/2 S1400; 3, phi2/2 Ex2.

diversity of plaque types was preserved for each phage-host pair. Possibly, in these cases, bacterial genomes contain defective or cryptic prophages related to the examined lytic phages, and their recombination occurs in the course of infection. This assumption has been directly confirmed by data obtained upon comparing restriction profiles of DNAs from phages phi135lar and phi135sm appearing in platings of single large plaques on strain Az269. Both of these variants were grown on strain Ex2, where no segregation occurs. As seen in Fig. 1, multiple crossovers occur when variant phi $135 \mathrm{sm}$ is formed. Thus, it is fully probable that phage phi135lar is a nonlysogenic (virulent) variant of the defective/cryptic prophage in strain Az269.

There is another observation of fundamental significance. It was detected that DNA extracted from preparations of some phages, which were obtained on 
the similar bacterial host, showed upon restriction an unusual pattern: many minor fragments of different molecular sizes appear in the background of the definite major bands where DNA of infecting phages undergoes fragmentation. Their number and sizes were found to vary in different species. It was assumed that these multiple minor fragments emerge after infection of cells from the same strain with various infecting phages due to the induction of many defective prophages and their packaging in capsids of bacterial DNA. This packaging protects DNA against the effect of DNase that is employed to prepare phage samples for DNA isolation. Our data obtained in the experiment with bacteriophage phi12 support this assumption (Fig. 3). A high-titer phage phi12 (preparation phil2ht) was purified and concentrated by mans of $\mathrm{PEG} / \mathrm{NaCl}$ [11] and was then treated with DNase. After phage precipitation (to remove the enzyme), DNA was extracted from a portion of resuspended phi12ht preparation. Another portion of the preparation underwent centrifugation in the $\mathrm{CsCl}$ density gradient, and DNA was isolated from a single band visible in the density gradient. As may be seen, the greater part of restriction fragments detected in DNA of phil2ht preparation prior to centrifugation in the $\mathrm{CsCl}$ density gradient disappear in the DNA sample from the phage after centrifugation.

Infection of cells with lytic bacteriophages may cause induction of the SOS response and of defective cryptic prophage [13]. We cannot state in which capsids (synthesized under the ontrol of the genome of defective or infecting phage) packaging of bacterial DNA occurs. In any case, the capsid that contains bacterial DNA, would sustain precipitation, being a component of lysate, and manifest instability upon centrifugation in the $\mathrm{CsCl}$ density gradient.

The results obtained raised the question about a reason for the predominance of various species of temperate phages (including nonlysogenic or virulent variants) in phage potential of salmonella species as compared to the true virulent phages (incapable of lysogenizing bacteria under any conditions). Some specificities of salmonella evolution and natural habitats of these bacteria can provide an answer to this question. The main mechanism underlying the evolution of salmonella species is the lysogenic conversion resulting from migration of phages among various hosts, which ensures the diversity in a series of genome pathogenic loci and effective exchange between these loci among strains of various serovars [14-19]. Meanwhile, optimal conditions for the development of salmonella pathogenic strains are created in the mucous envelope of human intestines in which bacteria are protected against the effect of virulent phages. Possibly, this explains a relatively low frequency of the incidence of true virulent phages and wide distribution of temperate salmonella phages.

Thus, infection of cells in some salmonella strains with lytic bacteriophages causes trans-activation of

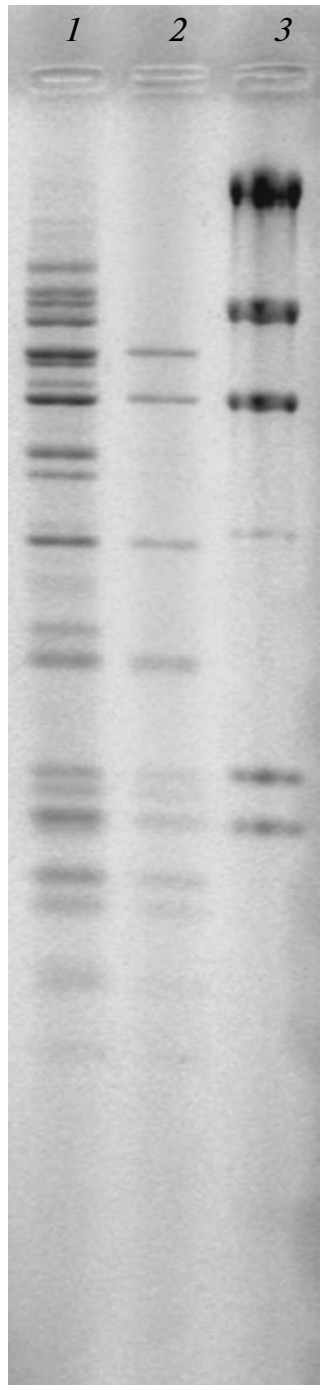

Fig. 3. Digestion with $E c o$ RV of DNA from phage phi12 before and after purification by centrifugation in the $\mathrm{CsCl}$ density gradient. 1, phi12ht, sedimented from the lisate; 2, phil2 after purification in the $\mathrm{CsCl}$ gradient; $3, \lambda$ HindIII (fragment size marker).

cryptic/defective prophages even in the absence of their direct relatedness with the infecting phage. Note that many free virions carrying variable regions of bacterial chromosome are formed in this case. While infecting bacteria of other strains, these virions, although they are not capable of self-replication, may constitute an essential proportion of the genetic elements that, like other integrons, participate in the distribution of genes responsible for multiple drug resistance or regions of islets of pathogenicity in bacterial genomes.

Collectively, these results suggest that the main direction of salmonella evolution mediated by migration of phages is an increase in the adaptive response to 
the intracellular growth, i.e., to the enhancement of pathogenicity.

\section{ACKNOWLEDGMENTS}

We thank the Profos AG Company (Bavaria) for the possibility to isolate a group of salmonella phages.

This work was supported by EC PhageVet-P (contract no. FOOD-CT-2005-007224) and by the Russian Foundation for Basic Research (grant no. 08-0400162-a). We gratefully acknowledge the support of organizations presenting the grants.

\section{REFERENCES}

1. Higgins, J.P., Andreatti Filho, R.L., Higgins, S.E., et al., Evaluation of Salmonella-Lytic Properties of Bacteriophages Isolated from Commercial Broiler Houses, Avian Dis., 2008, vol. 52, no. 1, pp. 139-142.

2. Atterbury, R.J., Van Bergen, M.A., Ortiz, F., et al., Bacteriophage Therapy to Reduce Salmonella Colonization of Broiler Chickens, Appl. Environ. Microbiol., 2007, vol. 73, no. 14, pp. 4543-4549.

3. O'Flynn, G., Coffey, A., Fitzgerald, G.F., and Ross, R.P., The Newly Isolated Lytic Bacteriophages st104a and st104b Are Highly Virulent against Salmonella enterica, J. Appl. Microbiol., 2006, vol. 101, no. 1, pp. 251-259.

4. Fiorentin, L., Vieira, N.D., and Barioni, W. Jr., Oral Treatment with Bacteriophages Reduces the Concentration of Salmonella enteritidis PT4 in Caecal Contents of Broilers, Avian Pathol., 2005, vol. 34, no. 3, pp. 258-263.

5. Krylov, V.N., The Role of Horizontal Gene Transfer by Bacteriophages in the Origin of Pathogenic Bacteria, Russ. J. Genet., 2003, vol. 39, no. 5, pp. 595-620.

6. San Martin, B., Lapierre, L., Cornejo, J., and Bucarey, S., Characterization of Antibiotic Resistance Genes Linked to Class 1 and 2 Integrons in Strains of Salmonella spp. Isolates from Swine, Can. J. Microbiol., 2008, vol. 54, no. 7, pp. 569-576.

7. Michael, G.B., Cardoso, M., and Schwarz, S., Molecular Analysis of Multiresistant Porcine Salmonella enteric subsp. enterica serovar Bredeney Isolates from Southern Brazil: Identification of Resistance Genes, Integrons and a Group II Intron, Int. J. Antimicrob. Agents, 2008, vol. 32, no. 2, pp. 120-129.
8. Martínez, N., Mendoza, M.C., Rodríguez, I., et al., Detailed Structure of Integrons and Transposons Carried by Large Conjugative Plasmids Responsible for Multidrug Resistance in Diverse Genomic Types of Salmonella enteric serovar Brandenburg, Antimicrob. Chemother., 2007, vol. 60, no. 6, pp. 1227-1234.

9. Miller, J.H., Experiments in Molecular Genetics, Cold Spring Harbor: Cold Spring Harbor Lab., 1972.

10. Adams, M.H., Bacteriophages, New York: Interscience, 1959.

11. Sambrook, J., Fritsch, E.F., and Maniatis, T., Molecular Cloning: A Laboratory Manual, Cold Spring Harbor: Cold Spring Harbor Lab., 1989.

12. Kropinski, A.M., Sulakvelidze, A., Konczy, P., and Poppe, C., Salmonella Phages and ProphagesGenomics and Practical Aspects, Methods Mol. Biol., 2007, vol. 394, pp. 367-380.

13. Downs, D.M. and Roth, J.R., A Novel P22 Prophage in Salmonella typhimurium, Genetics, 1987, vol. 117, no. 3, pp. 367-380.

14. Figueroa-Bossi, N., Coissac, E., Netter, P., and Bossi, L., Unsuspected Prophage-Like Elements in Salmonella typhimurium, Mol. Microbiol., 1997, vol. 25, no. 1, pp. 161-173.

15. Schmieger, H., Molecular Survey of the Salmonella Phage Typing System of Anderson, J. Bacteriol., 1999, vol. 181 , no. 5, pp. 1630-1635.

16. Rabsch, W., Mirold, S., Hardt, W.D., and Tschäpe, H., The Dual Role of Wild Phages for Horizontal Gene Transfer among Salmonella Strains, Munch Tierarztl. Wochenschr., 2002, vol. 115, nos. 9-10, pp. 355-359.

17. Figueroa-Bossi, N. and Bossi, L.J., Resuscitation of a Defective Prophage in Salmonella Cocultures, Bacteriol., 2004, vol. 186, no. 12, pp. 4038-4041.

18. Figueroa-Bossi, N., Uzzau, S., Maloriol, D., and Bossi, L., Variable Assortment of Prophages Provides a Transferable Repertoire of Pathogenic Determinants in Salmonella, Mol. Microbiol., 2001, vol. 39, no. 2, pp. 260-271.

19. Schicklmaier, P., Moser, E., Wieland, T., et al., A Comparative Study on the Frequency of Prophages among Natural Isolates of Salmonella and Escherichia coli with Emphasis on Generalized Transducers, Antonie Van Leeuwenhoek, 1998, vol. 73, pp. 49-54. 